\title{
Proofreading familiar text: Allocating resources to perceptual and conceptual processes
}

\author{
BETTY ANN LEVY and JOHN BEGIN \\ McMaster University, Hamilton, Ontario, Canada
}

\begin{abstract}
Levy (1983) demonstrated that more spelling errors were detected, within a limited time period, when familiar passages were proofread than when unfamiliar passages were proofread. In the present series, Experiment 1 eliminated a possible confound in the Levy (1983) studies and showed that errors were detected both faster and more accurately in familiar texts. Experiment 2 demonstrated higher order involvement in the proofreading transfer effect, suggesting that a strictly word-level account was insufficient. Experiment 3 explored the proofreader's sensitivity to the semantic properties of the proofreading passage, showing that the familiarity effect resulted from more efficient processing, not from lack of either visual or semantic analyses. The results are more consistent with a resource-allocation explanation than with either a visual-scanning or a skilled-visual-processing account.
\end{abstract}

The theoretical issue addressed in the present experiments was the relationship between familiarity with a text and the processing of the written words, with their constituent letters, within that text. Familiarity may lead to faster, more fluent rereading of a passage, but the question is whether a gain in rereading speed is through less thorough analysis of the printed display. That is, reading speed may be gained because less visual processing is needed to recognize familiar word patterns, and thus the printed text is less completely analyzed during rereading. Or, rapid rereading may include just as thorough, but more efficient, analysis of the visual display. Thus, speed may be gained either through attenuated or through more fluent visual reprocessing of a familiar text. Our interest, then, was in the relationship between familiarity and the extensiveness of visual word and letter analyses.

Many earlier studies have used constituent-letterdetection tasks to investigate the relationship between word familiarity and constituent-letter recognition. These studies frequently have reported a word superiority effect (Reicher, 1969; Wheeler, 1970), whereby the perception of a target letter is facilitated because the letter is embedded in a familiar word, rather than in a nonword. These studies suggest that familiarity with a word unit

This research was supported by Grant A7657 from the Natural Sciences and Engineering Research Council of Canada. The manuscript was prepared while the first author was on sabbatical leave visiting the School of Behavioural Sciences, Macquarie University, Sydney, Australia, and the Department of Psychology, Monash University, Melbourne, Australia. We thank colleagues there for helpful discussions of the work reported here, and particularly Adrienne Bennett, Steven Hausfeld, and Donald Thomson for their comments on drafts of the manuscript. We also thank Meredyth Daneman for providing most of the passages used in Experiment 3 and for her critical comments on the Levy (1983) paper that motivated some of the issues studied here. The authors' mailing address is: Department of Psychology, McMaster University, Hamilton, Ontario, Canada L8S 4K1. facilitates identification of its constituent letters. It is unclear, however, whether this word superiority effect generalizes from single-word recognition to text-reading tasks. Healy and Drewnowski (1983), using a constituentletter-detection task, recently reported a word inferiority effect in text reading. They explain the discrepancy between the word superiority effect in word recognition and the word inferiority effect in text reading by suggesting that processing resources are allocated differently in the two tasks. While reading fluently, subjects quickly recognize a word and then move on to the next word, without fully analyzing the letters of the recognized word. However, in a tachistoscopic word-recognition task, all processing resources are necessarily devoted to that single word, so that letter analyses may occur after word recognition itself. By this view, there is no automatic feedback from word to constituent-letter analyses, so that full letter analyses may occur only when resources are not required for other processing.

From the constituent-letter-detection tasks, it remains unclear whether familiarity with the words of a text will facilitate or hinder letter-level processing. However, using a proofreading task, Levy (1983) provided some evidence that familiarity can facilitate letter-level processing. Proofreading, of course, requires detection of an unexpected or deviant element, rather than of a constituent element, of a familiar word. This feature of the task makes it an interesting method for pursuing the relationship between familiarity and letter processing, since deviant letters cannot be guessed from higher level information. Thus, error detection may provide a clearer indicator of the extensiveness of letter processing from the written text than does constituent-letter-detection.

The present studies pursued further the familiarity benefit in proofreading reported by Levy (1983). In that study, she found that subjects detected more spelling errors in familiar than in unfamiliar passages. Familiarity 
was manipulated by having subjects read an error-free version of the passage prior to performing the proofreading task. Within a limited time period, proofreaders found more misspellings in passages that they had read previously than they did in novel passages. Furthermore, this facilitation in proofreading following reading was equivalent whether the words on the proofreading page were in the original text order or in a scrambled arrangement. Thus, the removal of the syntactic-semantic component of the original reading experience did not diminish the transfer benefit from reading to proofreading. This lack of higher order dependency suggested a word- or letterlevel interpretation of the familiarity benefit. Furthermore, preserving the meaning, but altering the wording and syntax (paraphrasing), or preserving the text, but altering the modality of presentation or the typescript, led to a decrease in the transfer benefit from reading to proofreading, to the level of nonspecific transfer for an unrelated passage. Thus, meaning preservation seemed to be unrelated to the transfer benefit, but repetition of the visual configurations was important to the positive effect. These lower level constraints, in the absence of syntactic-semantic mediation, led Levy (1983) to conclude that the facilitation was mediated by word- or letter-level processes.

The experiments to be described addressed several further issues regarding the transfer benefit observed in proofreading. The first two experiments demonstrated that (1) there is a gain in reprocessing fluency of familiarized texts in a proofreading task and (2) this reprocessing gain is not due simply to a reduction in the amount of visual letter processing. To address these issues, a self-paced procedure, rather than the time-limited task used by Levy (1983), was adopted. This procedure allowed us to examine the effects of familiarization on both rereading speed and visual error-detection accuracy, thus eliminating an interpretative problem found in the 1983 study. Experiment 2 also explored the involvement of higher level processes in the transfer from reading to proofreading, by looking at the influence of prior experience with a wellorganized text versus experience with only the words to appear on the proofreading page.

Since Experiments 1 and 2 had established that the reprocessing gain is not through reduced visual processing, Experiment 3 then asked whether the processing gain could be due to the elimination of semantic processing during proofreading. That is, could proofreading speed be gained by visual scanning of the printed array for nonlexical letter combinations, without in fact reading the proofreading text at the message-comprehension level? Thus, speed gained in reprocessing familiar text would be due to the elimination of comprehension of the proofread message.

Finally, the general discussion focuses on the levels of processing and the possible mechanisms involved in the proofreading facilitation, as indicated by the data from all studies. In total, then, the experiments extended the phenomenon described by Levy (1983), and attempted to bring some closure on the possible mechanisms involved in the transfer effect. The manipulations and hypotheses relevant to each study will be described prior to that experiment.

\section{EXPERIMENT 1}

In the study reported by Levy (1983), proofreading time had been limited to $1.5 \mathrm{~min}$, and the dependent variable was the number of errors found in that time period. The greater detection accuracy observed for familiar than for unfamiliar passages might have been related to the increased perceptibility of errors in familiar passages, or it might have been related to improved reading speed (fluency) through familiar texts. In the fixed-time procedure, since subjects could undoubtedly read familiar texts faster than they could unfamiliar ones, it is possible that only familiar passages were completely read within the 1.5-min time limit. If unfamiliar passages were not read entirely in $1.5 \mathrm{~min}$, there would be a decreased probability of encountering the errors at the end of the passage. Thus, detection accuracy in familiar versus unfamiliar passages was potentially confounded with the amount of the passage read in $1.5 \mathrm{~min}$ of proofreading. Since no measure was taken of the amount of the passage proofread, this possibility could not be examined. In order to ensure that the entire text was proofread, whether familiar or unfamiliar to the proofreader, Experiment 1 adopted a selfpaced procedure. The experiment was basically a replication of the original studies, but the subjects were told to proofread the passage as quickly and accurately as possible, and then both proofreading speed and accuracy were used as dependent measures. This procedure examines the effect of familiarization on rereading speed, as well as on visual processing through error-detection accuracy.

Although the self-paced procedure overcomes the potential confounding in the Levy (1983) time-limited procedure, it also introduces the possibility of a time-accuracy trade-off. The basic question pursued in Experiments 1 and 2 here was whether prior familiarity with a text leads to faster rereading because readers fail to fully analyze the visual display on the later reading encounter. That is, the fluency gained in rereading is through less thorough analysis of the written materials. The proofreading task, then, gauges the visual analyses performed during reading, whereas proofreading speed indicates the rereading fluency benefit.

Since proofreaders, in the self-paced procedure, are free to vary either proofreading speed or accuracy, the facilitation in performance due to familiarity may be observed in either or both measures. That is, proofreaders may set an accuracy criterion and vary proofreading speed to meet that criterion, or they may set a speed criterion and vary detection accuracy. A loss in visual processing would be indicated by a reduction in error-detection accuracy, whereas more efficient reprocessing would be shown by faster rereading with no loss in visual error detection. Better error detection with faster or comparable reading speed would indicate improved perceptibility, with improved or 
equivalent rereading fluency. Although the accuracy effects are more critical to the visual-processing questions, speed changes are informative for questions of reprocessing fluency at all levels of processing. The speed-accuracy relationships should tell us something about the allocation of resources during rereading to meet task demands.

Experiment 1, then, replicated Levy's (1983) studies, using a self-paced procedure. Instructions to stress speed versus accuracy were used to examine shifts due to the emphasis in the task. As in the earlier studies, both easy and difficult texts were used to examine the generality of the effects observed to different populations of texts. Also, transfer from reading to proofreading was measured for passages proofread in the original text arrangement or in a scrambled arrangement, since the equivalence of facilitation in these two conditions had been used to localize the transfer benefit at the word or letter levels. Facilitation in proofreading due to prior reading experience was expected for both normal and scrambled proofreading tasks, and irrespective of the difficulty of the text being read.

\section{Method}

Materials and Design. The experimental task required proofreaders to read through each passage once only, striking out any word they thought contained a spelling error. The experimental design contained one between-subjects variable and three withinsubject variables. The between-subjects manipulation was the instruction concerning proofreading speed and accuracy. Half of the subjects formed a speed-stress group, whereas the other half formed an accuracy-stress group. Instructions for the two groups differed in only one sentence. For the speed-stress group, this sentence read, "The important thing is to read as quickly as you can, since we will be timing your reading speed, but still slashing out as many misspellings as you see." For the accuracy-stress group, this sentence was changed to read, "The important thing is to read carefully so you slash out as many misspellings as possible, but yet reading as quickly as you can, since we will be timing your reading speed." In the summaries at the end of each set of instructions, there was also a reminder to read quickly or read accurately, as appropriate to that group. Other than this instructional variation, both groups of subjects were treated identically.

The first within-subjects manipulation consisted of the type of passage to be proofread. All passages were exactly 350 words in length, being length-modified versions of the easy and difficult materials described by Levy (1983). The easy passages were taken from the intermediate levels of the SRA reading series, and the difficult passages came from senior-level psychology textbooks. These passages differed not only in text structure, but also in word length, frequency, concreteness, etc. A fuller description and sample passages can be found in Levy (1983). Each subject proofread four easy and four difficult passages. There were two sets of such materials, each read by half of the subjects in both groups.

Three versions of each of these eight passages per set were constructed. One version was a normally arranged error-free text used only for the familiarization training. The two remaining versions were for proofreading tests only. Each of these versions contained exactly the same 24 misspelled words, in exactly the same text positions on the page. The two versions differed in the arrangement of the contextual words. For the normal version, the text was identical to the familiarization passage, except for the 24 error words. For the scrambled version, all of the words surrounding the $24 \mathrm{er}-$ ror words were rearranged randomly. The scrambling was not within sentences, but across the entire text, so that no syntactic constraints of the original sentences remained. These random words were then organized into pseudosentences and pseudoparagraphs by insertion of punctuation marks and paragraph spacing in the same places in which they had occurred in the normal text. Thus, the normal and scrambled passages looked similar, with the error words in the same locations, but in the normal case the original syntactic-semantic constraints were maintained, whereas in the scrambled case these were violated. Each of the subjects proofread two easy and two difficult passages in their normal versions and two each in their scrambled versions.

The third within-subjects variable was reading experience before the proofreading task. This prior reading was always of a normally arranged text. For each of the subjects, half of the passages were proofread without any prior reading experience, whereas for the other half of the passages, the subjects read the error-free version aloud, three times, immediately prior to proofreading that passage. The subjects were told that knowing the passage meaning would help them to find the spelling errors, even when the proofreading page was a scrambled version of the one they had just read.

The orthogonal combination of these three within-subjects variables thus determined the eight proofreading conditions. For each subject, one normal text and one scrambled text were proofread without prior reading, and one of each was proofread following reading of the normal text. These four conditions were tested once with easy and once with difficult passages. The eight passages per set were circulated through the conditions, so that no materials differences existed for either instruction group, for the four basic conditions tested with either easy or difficult materials.

The spelling errors used in the proofreading task were identical for the normal and scrambled versions of each passage. The 24 spelling errors per passage occurred randomly within the text, with the constraints that the errors be approximately equally frequent in each half of each text and that 6 errors occur in each of four syntactic classes (function words, nouns, verbs, and adjectives). For the last three classes, the errors occurred equally often in first and later syllables. As in Levy (1983), all error substitution letters were chosen to (1) maintain the original word shape, (2) change the word into a nonword, and (3) have no acoustic similarity to the target letter. Exactly the same error-filled sheets were used for familiarized and unfamiliarized conditions.

In summary, then, the subjects proofread four easy and four difficult passages, each containing 24 spelling errors, in familiar passages that were normally arranged or in familiar texts that had been scrambled for proofreading, and in unfamiliar passages that were in a normal or a scrambled arrangement. The data of interest were the numbers of errors detected and the proofreading times, for one group of subjects given speed-stress instuctions and one group given accuracy-stress instructions.

Procedure. The subjects were assigned randomly to the speed or accuracy groups as they arrived in the laboratory for their individual 45-min sessions. After receiving the appropriate set of instructions, the subjects were given one practice passage to familiarize them with the experimental task and then were given the eight experimental passages. To measure proofreading time, a stopwatch was started as the proofreading page was turned over in front of a subject and was stopped when the subject raised his(her) hand to indicate completion of the task. The subjects were not told in advance whether they would next receive a normal or a scrambled version for proofreading, and although they knew that the passage contained spelling errors, no information was given on the number or placement of these errors.

Subjects. Sixty-four undergraduate volunteers ( 32 in each instruction group) participated in this study. All of the students were registered in an introductory psychology class and received course credit for their participation. All participants were native English speakers and were less than 35 years of age. 


\section{Results and Discussion}

Proofreading accuracy. The self-paced procedure used here led to higher overall detection accuracy than the timelimited procedure used by Levy (1983), but no ceiling effects were evident in the data. Also, since the proportion of correctly spelled words erroneously slashed as misspellings was approximately zero $(.000006)$, false-alarm rates need not be considered here or in subsequent experiments, in which, again, the false-alarm rate was approximately zero. Finally, since the main manipulations did not interact with the syntactic class of the error word, the accuracy data were combined across syntactic class and were subjected to a mixed-design analysis of variance. Instructions (speed vs. accuracy) was a groups variable, and proofreading version (normal vs. scrambled), familiarity (familiarized vs. unfamiliarized), and passage difficulty (easy vs. difficult) were repeated measures. The main effect of instructional set was not significant $(F<1)$, and this variable entered into no interactions. Two main effects were significant, that of familiarization $[F(1,62)=$ $52.18, \mathrm{p}<.0001]$, indicating that more errors were detected in familiar than in unfamiliar passages, and that of passage difficulty $[F(1,62)=7.95, p<.007]$, showing better detection of errors in difficult than in easy passages. Table 1 contains the means relevant to these effects.

The only other effect to approach significance was the interaction of version, familiarity, and passage difficulty $[F(1,62)=3.64, p<.058]$. The only critical point to note is that this trend does not stem from differential familiarity effects in the normal versus the scrambled conditions, but seems to indicate differential tendencies for proofreading to be better in difficult than in easy passages, across the four version $\times$ familiarity conditions. To summarize, two findings of Levy (1983) were replicated using the self-paced procedure. More errors were detected in familiar than in unfamiliar passages, and this familiarization benefit was equivalent whether transfer was from reading a normal text to proofreading that same text or from reading a normal text to proofreading a scrambled version of that text. Again, the locus of the familiarization benefit appeared to be at the letter or word levels.

Proofreading times. The times (in seconds) taken to proofread each passage were subjected to the same mixeddesign analysis of variance used to analyze the accuracy data. Again, there was no main effect of instruction group $(F<1)$, and the group factor entered into no interactions. Thus, the speed versus accuracy stress given in the in- structions affected neither the time taken to proofread nor the accuracy in finding the misspellings. All three withinsubjects variables influenced proofreading times ( $p<.0001$ in each case). Familiar passages were proofread faster than unfamiliar passages $[\mathrm{F}(1,62)=$ 29.44]; normal passages were proofread faster than scrambled passages $[\mathrm{F}(1,62)=55.82]$; and easy passages were proofread faster than difficult passages $[\mathrm{F}(1,62)=$ 80.04]. Besides these main effects, the interaction of version with familiarity was also significant $[F(1,62)=$ $14.85, \mathrm{p}<.0005]$. No other interactions approached significance. As Table 1 clearly indicates, this version $x$ familiarity interaction stems from the larger familiarization benefit for normal than for scrambled passages. The decrease in proofreading time for familiar versus unfamiliar passages missed significance for the scrambled texts $[t(63)=1.98, p<.10]$, but was highly reliable for the normal texts $[\mathrm{t}(63)=18.12, \mathrm{p}<.0001]$.

For the time and accuracy data combined, several points should be noted. The data for easy and difficult passages show a time-accuracy trade-off, whereby difficult passages were proofread better but more slowly than easy passages. This trade-off may have been instrumental in the interaction involving passage type in the accuracy data, and makes further interpretation of this materials effect unwise. Importantly, the familiarity effect was consistent for both easy and difficult materials.

This speed-accuracy trade-off problem was not observed for any of the critical familiarity comparisons. Familiar texts were proofread both faster and more accurately than unfamiliar texts. Thus, the increased speed observed in rereading familiar texts was not gained through less thorough analysis of the written pages, as indicated by the accuracy in detecting spelling errors within these familiar words. These data replicate the familiarity effect reported by Levy (1983), indicating that the potential confounding involved in the time-limited procedure was not instrumental in causing the improved proofreading of familiar passages.

The second finding of importance is less clear-cut. Whereas the accuracy data support Levy's (1983) finding of equal transfer from reading a normal text to proofreading either a normal or a scrambled version of that text, the proofreading time data show unequal transfer. Here, scrambled texts show less familiarity facilitation in rereading time than do normal texts. This time difference is not related to a time-accuracy trade-off, since both scrambled and normal texts show a familiarity benefit

Table 1

Proofreading Accuracy (Mean Proportion Detected) and Times (Means in Seconds) for Experiment 1

\begin{tabular}{llccccc}
\hline & & \multicolumn{2}{c}{ Familiarized (Normal) } & & \multicolumn{2}{c}{ Unfamiliarized } \\
\cline { 3 - 3 } Easy Texts & Accuracy & Time & & Accuracy & Time \\
\hline \multirow{2}{*}{ Difficult Texts } & Proofread Normal & .86 & 102.10 & & .83 & 111.12 \\
& Proofread Scrambled & .87 & 126.09 & & .81 & 130.89 \\
& Proofread Normal & .88 & 114.84 & & .83 & 123.95 \\
& Proofread Scrambled & .88 & 141.76 & & .84 & 142.79 \\
\hline
\end{tabular}


in both time and accuracy measures. Although the equality of effects in the accuracy data suggests a localization of the transfer benefit at the word or letter levels of analysis, the time difference suggests that the loss of syntacticsemantic constraints from reading to proofreading texts may lead to less transfer than when these higher levels are also repeated. Since this issue is addressed again in Experiment 2, further discussion of the specificity of transfer will be taken up there. For now, it can be concluded that familiarity leads to more fluent (faster) rereading of a text, without loss of thorough visual analysis of the written words or letters within that text.

\section{EXPERIMENT 2}

Experiment 2 tested the dependence of the proofreading facilitation on having first experienced a wellorganized text, compared with having experienced only the visual word patterns during original reading. In Experiment 1 , and in the studies reported by Levy (1983), the reading passages used for familiarization prior to proofreading had always been in a normal text arrangement. Original reading of a well-organized text may set up a text representation that can be used to more rapidly re-encode even a scrambled version of that text. Thus, the higher order syntactic-semantic organization of the original reading text may aid in proofreading, even for the scrambled task, indicating a dependence on higher level information from the original reading task. If such higher order involvement were critical to the occurrence of transfer from reading to proofreading, then no transfer should be observed when the first reading experience was of a scrambled text.

Experiment 2, then, explored further the involvement of syntactic-semantic processes by asking subjects first to read either a normal or a scrambled text and then to proofread either the normal or the scrambled version of that text. This combination yielded four conditions, normal reading followed by either normal or scrambled proofreading, and scrambled reading followed by normal or scrambled proofreading. The critical point here is that at the level of word repetition between reading and proofreading tasks, the four conditions are equivalent. However, at the level of syntactic-semantic organization, the scrambled reading conditions do not provide a higher order text organization to aid in the subsequent proofreading task. If transfer is mediated entirely at the word level, then the syntactic-semantic organization of the passages should not influence the familiarity benefit observed in proofreading. On the other hand, if higher order processes play some role in the transfer to proofreading, then scrambled reading conditions should alter the familiarity effect.

\section{Method}

The experimental task was like that described in Experiment 1, using the speed-stress instructions. Each of the subjects proofread the eight easy passages used in Experiment 1. The same scrambled versions and the same error words described there were used in this study. The data of interest were the number of errors detected and the time taken to proofread.

Four versions of each of the eight passages were created, a normal and a scrambled version without errors, and the same normal and scrambled versions with errors. Each of the subjects read one of the eight passages in each of the four familiarized conditions: (1) familiarized on normal, proofread normal; (2) familiarized on normal, proofread scrambled; (3) familiarized on scrambled, proofread scrambled; and (4) familiarized on scrambled, proofread normal. In all cases, the familiarization consisted of three oral readings of the error-free training passage. The remaining four passages were proofread without prior reading experience, two proofread in the normal version and two in the scrambled version. The passages were circulated through the eight testing conditions to ensure that each passage was tested equally often in each condition, across subjects. Thus, again there were no materials confoundings across comparisons.

The subjects were tested individually, in 45-min sessions. One practice passage preceded the experimental trials. Instructions and timing were as in Experiment 1. Thirty-two different student volunteers from the population described there participated in this study.

\section{Results and Discussion}

Proofreading accuracy. Analysis of variance of the accuracy data revealed only one main effect. Misspellings were better detected in familiar than in unfamiliar texts $[F(1,32)=8.55, p<.01]$, replicating the familiarity effect. There was no tendency for the magnitude of the familiarity benefit to vary with the transfer variations (see Table 2). However, this accuracy equivalence across syntactic variations was maintained by changing the proofreading times, as indicated by the analyses below.

Proofreading times. For each of the subjects, the mean proofreading times were taken for the two passages proofread in the normal-unfamiliar and for the two proofread in the scrambled-unfamiliar conditions, after having determined that there were no significant differences between these tests. Thus, the data submitted to analysis of variance were the reading times (in seconds) for the 2 proofreading conditions (normal, scrambled) $\times$ the 3 familiarization conditions (familiarized-normal, familiarized-scrambled, unfamiliarized). Both main effects and the interaction were significant beyond the $\mathrm{p}<.0001$

Table 2

Proofreading Accuracy (Mean Proportion Detected), Times (Means in Seconds), and Mean Time Savings (UF-F), for Experiment 2

Proofreading Accuracy and Times

\begin{tabular}{|c|c|c|c|c|c|c|c|c|}
\hline & \multirow{2}{*}{\multicolumn{2}{|c|}{ Familiarized-Normal }} & & & & & \multirow{2}{*}{\multicolumn{2}{|c|}{ Mean Time Savings }} \\
\hline & & & \multicolumn{2}{|c|}{ Familiarized-Scrambled } & \multicolumn{2}{|c|}{ Unfamiliarized } & & \\
\hline & Accuracy & Time & Accuracy & Time & Accuracy & Time & Familiarized-Normal & Familiarized-Scrambled \\
\hline Proofread-Normal & .84 & 89.29 & .82 & 95.27 & .79 & 97.24 & 7.95 & 1.98 \\
\hline Proofread-Scrambled & .82 & 105.63 & .83 & 98.28 & .80 & 110.81 & 5.18 & 12.53 \\
\hline
\end{tabular}


level. Normal passages were proofread faster than scrambled passages $[\mathrm{F}(1,31)=53.44]$, and the familiarization condition led to faster proofreading than did the unfamiliar condition $[F(2,62)=16.48]$. The interaction $[F(2,62)=$ 15.56] stems from the differential magnitude of the familiarity facilitation for the four transfer conditions. Table 2 contains the proofreading times for the interaction.

In order to clarify this differential familiarity effect, a savings score was calculated for each subject, for each of the four familiarization conditions. The savings score consisted of the unfamiliarized minus the familiarized proofreading times for each condition, and thus represented the savings in proofreading times due to familiarization. These individual savings scores were then submitted to analysis of variance, in which the variables were proofreading condition (normal, scrambled) and reading experience (normal, scrambled). Although both main effects missed significance, the critical interaction was highly significant $[\mathrm{F}(1,31)=26.00, \mathrm{p}<.0001]$. Table 2 shows the mean savings for the four conditions.

Comparison of transfer following familiarization with a normal text shows the usual familiarization transfer effect for proofreading both normal and scrambled versions of that text. Whereas in Experiment 1 there had been significantly more facilitation in proofreading time for the normal-normal than for the normal-scrambled condition, this trend did not reach significance here $[t(1,31)=1.06]$. Following familiarization with a scrambled text, however, proofreading time savings were not equivalent for the normal and scrambled proofreading versions of that passage. As Table 2 indicates, substantial improvement in proofreading speed occurred when the proofreading page was also scrambled $(12.5 \mathrm{sec})$, but little benefit occurred when the proofreading page was in a normal arrangement $(1.98 \mathrm{sec})$. Thus, reprocessing fluency, as gauged by proofreading times, was not equivalent for the two text versions, when the prior reading experience had not familiarized the proofreader with the syntactic and semantic aspects of that text. Finally, the trend for the scrambled-scrambled condition to show more transfer than the normal-normal condition was not significant $[\mathrm{t}(31)=1.87]$.

For the proofreading time and accuracy data combined, then, several points should be noted. Three of the experimental conditions (normal-normal, scrambledscrambled, normal-scrambled) replicated the findings of Experiment 1, showing that fluency gained in reprocessing a familiar passage was not due to less thorough analysis of the visual display, as indicated by the superior error-detection accuracy. For the scrambled-normal condition, however, the improved proofreading accuracy for familiar passages was coupled with little improvement in proofreading speed. There was an overall familiarity benefit, even in this condition, but its magnitude was markedly smaller (and localized largely in accuracy) than those found in the other familiarized conditions.

The results of Experiment 2 show that the magnitude of the familiarization benefit varies with the syntacticsemantic overlap between the original and proofreading texts. Thus, whereas the normal reading conditions from this experiment suggested that the transfer effect occurred largely at the word or letter level of analysis, the scrambled reading conditions indicated the involvement of higher level processes. This involvement, however, is not of the simple nature that a syntactic-semantic text representation from the original reading experience is essential for the transfer to occur, since the largest transfer effect is in the scrambled-scrambled condition, in which no text representation is involved either in reading or in proofreading. Rather, structural involvement is related to the degree of familiarity with that structure, when it recurs in the proofreading task. When a normal text is proofread, familiarity with that text structure yields a familiarity benefit (normal-normal) that decreases dramatically when the proofreader is unfamiliar (scrambled-normal) with that text structure (time savings $=7.95$ and $1.98 \mathrm{sec}$, respectively) $[\mathrm{t}(31)=3.38, \mathrm{p}<.01]$.

Although this difference due to familiarity with the text structure of normal texts might suggest that the prior representation of the syntactic-semantic structure in some way "guided" the later reprocessing for visual errors, this notion is seriously weakened by a similar effect occurring for scrambled texts. When a scrambled passage is proofread, it is also better to have previously read that scrambled version (scrambled-scrambled) than to have previously read a normal version (normal-scrambled) of that text (time savings $=12.53$ and $5.18 \mathrm{sec}$, respectively) $[t(31)=3.53, p<.01]$. Here it is inappropriate to suggest that higher order syntactic-semantic processes "guide" later proofreading. Rather, when the structural aspects of the texts differ from reading to proofreading tasks, the magnitude of the familiarization benefit decreases, suggesting that additional processing is required for rereading the new structural arrangement. Whereas the accuracy effects in the present experiment could be accounted for by improved perceptibility at the word or letter level, the reprocessing fluency effects necessitate higher order involvement in any complete explanation of the familiarity benefit. These results are also inconsistent with a notion that syntactic-semantic processes "guide" later error detection through that passage, although they suggest that structural familiarity aids rereading speed.

\section{EXPERIMENT 3}

Our work on the proofreading paradigm was originally motivated by an interest in the relationship between the fluency gains in rereading speed through familiar passages and the amount of visual analysis given to the written words and letters within those texts. Experiments 1 and 2 demonstrated that the faster rereading of familiar texts occurred through more efficient reprocessing, not through 
less thorough analysis of the written configurations. Familiar passages were proofread both faster and more accurately in both experiments. Furthermore, Experiment 2 indicated that reprocessing speed gains due to familiarization were influenced by the structural arrangement of the passages. Experiment 3 explored further the involvement of higher order processing in this reprocessing benefit.

In the previous two experiments, we assumed that the subjects followed our instructions to "read" the proofreading passages, although no measure of message comprehension during proofreading was taken. However, an alternative possibility is that the subjects allocated no processing resources to syntactic-semantic analyses of a "known" message. Rather, they scanned the visual display, detecting error words through failure to find a lexical entry for the nonword letter combinations used to form the misspelled words. Thus, one explanation for the rapid rereading and superior error detection for familiar texts is that visual scanning is faster than reading, so speed was gained through failure to analyze the passage's message, thus concentrating all resources on improving error detection.

A reprocessing-efficiency view, on the other hand, would suggest that subjects do analyze the familiar passages for meaning, but that this reprocessing is done more efficiently. The idea is that the representation of the message formed during original reading allows more efficient reprocessing, much as "schema" allows easier comprehension of a familiar idea. This more efficient reprocessing frees resources to improve performance on the error-detection task. Thus, the familiarity benefit is not through failure to engage higher order processes, but rather through more efficient, less resource-demanding, semantic monitoring of the "known" message.

Experiment 3, therefore, explored the proofreaders' sensitivity to the message being proofread. The problem, of course, was how to gauge sensitivity during proofreading, since the subjects were already familiar with the text from the reading task, thus making a simple recall or recognition test inappropriate. In Experiment 3, we varied the similarity between the reading and proofreading texts, thus allowing us to measure semantic sensitivity in terms of the disruptions in proofreading speed and accuracy when the message was altered from the reading to the proofreading tasks. The idea was that if subjects who are familiar with a passage "turn off" higher level analyses, as the scanning view suggests, they should be impervious to deviations between the reading and proofreading texts. By the "efficiency" view, however, since the proofreading message is being monitored for similarity with the known message, serious semantic alterations should be noted. When the semantic processor "defaults," signaling a comprehension failure, resources should be reallocated from the proofreading task to the semantic processor, thus causing a failure in fluency in the proofreading task. Thus, when the passage contains a semantic default, semantic sensitivity should be detectable through a loss in proofreading performance.

In a pilot study, we examined single word changes that did not disturb the sense of the proofreading message and found that the semantic monitoring process was insensitive to such unimportant word changes. In Experiment 3, therefore, the semantic changes made on the proofreading pages altered the sense of the target sentence and led to an inappropriate interpretation. If subjects are simply scanning for nonwords, this change should go unnoticed. However, if the comprehension of the message is being monitored, these sense changes should cause a default in the semantic processor and thus slow reprocessing and take attention from the proofreading task, therefore causing a decrease in proofreading performance. This sensitivity to semantic distortion on the proofreading page was measured for both familiar and unfamiliar passages. The questions of interest were (1) would subjects show sensitivity to the semantic defaults during proofreading, and (2) would this sensitivity be less for familiar than for unfamiliar passages? Finally, a recognition memory test for the phrase causing the semantic problem followed each proofreading trial. This measure was taken as another potential indicator of semantic processing during proofreading.

\section{Method}

The subjects proofread eight pages, each containing two 100-word paragraphs. The two paragraphs per page were semantically unrelated, but were combined to form a test "page." Both paragraphs on a page represented the same test condition and were treated as a single test trial, so that the "page" was the unit of analysis, as in the earlier studies. Each paragraph contained one "garden-path" sentence capable of being interpreted in two ways, because of an ambiguous word within it. The sentences prior to this garden-path sentence were biased toward one of its interpretations. The sentence following the garden-path sentence was then either semantically consistent or semantically inconsistent with this biased interpretation. The semantic inconsistency was caused by altering only a few words in the final clause of this subsequent sentence. The inconsistent version required reinterpretation of the garden-path statement, using the other meaning of the ambiguous word. Two types of ambiguous words were used: homographs that led to both pronunciation and semantic miscues (e.g., "tears"), and homonyms that led to only semantic miscues (e.g., "bat"). Since the homographic words caused more miscuing, it seemed reasonable to expect the disruptive effects here to be more detectable during proofreading. Table 3 contains examples of homograph and homonym passages. Of the eight test pages, four contained two 100-word homonym paragraphs and four contained two 100-word homograph paragraphs. For each page, there was an error-free semantically consistent version used for the reading phase, as well as an error-filled consistent and an error-filled inconsistent version used for proofreading.

Each of the 100-word paragraphs per page contained seven misspellings, all conforming to the criteria used in the earlier studies. Two errors occurred in function and five in content words. One content-word error was always in the garden-path sentence (although never in the ambiguous word), and two occurred in the sentence following the garden-path statement. The remaining two contentand the two function-word errors occurred in other sentences across 
the passage. Since there were two paragraphs per page, each proofreading page contained a total of 14 misspelled words. Consistent and inconsistent versions of each page contained identical errors, except for the one error located in each altered clause. Here the error was placed in approximately the same location for both versions, but was of course in a different content word.

For two homonym and two homograph trials, the subjects proofread without prior reading of the page, proofreading one of each type in its consistent and one in its inconsistent version. This consistent-inconsistent comparison allowed a measure of the detection of the garden-path default, when subjects were proofreading unfamiliar texts. The remaining two homonym and two homograph pages were proofread following familiarization. Familiarization always consisted of three oral readings of an errorfree consistent version of each passage. Thus, the subjects were never alerted to which was the garden-path sentence in each paragraph, during the original reading encounter. One familiarized homonym page and one familiarized homograph page were then proofread in each of the consistent and inconsistent versions. That is, the subjects proofread four homonym and four homograph pages, an unfamiliar-consistent, an unfamiliar-inconsistent, a familiarconsistent, and a familiar-inconsistent version. The four pages of each type were used equally often in each of the conditions across subjects, and order of conditions was also counterbalanced across subjects.

Following each proofreading trial, the subjects made two "yes"'"no" sentence-recognition judgments. The two statements tested were those following the two garden-path statements per page (i.e., the statements causing the semantic default; see Table 3 ). Two forms of the recognition test were used, such that one version of each statement was used on each test form, and for each test form half of the judgments required "yes" and half required "no" responses. The two test versions were used equally often for all conditions. The subjects were told that the familiarization and proofreading passages were similar, but not necessarily identical, and that the sentence-recognition test was for the statement on the proofreading, not the familiarization, page. Thus, comprehension during proofreading was encouraged, and the recognition test provided data on later memory for the materials proofread. To familiarize the subjects with the proofreading and recognition memory tasks, one practice trial preceded the eight experimental tests. No information on number or distribution of errors was given. Thirty-two undergraduate volunteers, from the pool already described, participated for course credit or for a $\$ 3.00$ payment.

\section{Results and Discussion}

The presentation of the proofreading data is complicated by the fact that the semantic default influenced proofread- ing somewhat differently for homonym versus homograph paragraphs. The homographic default, signaled by both a pronunciation and a semantic miscue, caused more processing disruption during proofreading than did the homonym paragraphs. Furthermore, paragraph type entered into three-way interactions with the familiarity and semantic-consistency effects for both the time and accuracy data $[F(1,31)=5.30, p<.03$, for accuracy, and $F(1,31)=11.81, p<.01$, for time]. Because of these differential effects for the two passage types, each type was separately analyzed for the influence of semantic consistency and familiarity.

Homonym paragraphs. Analyses of variance of the proofreading-time and accuracy data indicated sensitivity to the semantic-consistency manipulation in both measures. Semantically consistent passages were proofread faster $[\mathrm{F}(1,31)=9.42, \mathrm{p}<.01]$ and more accurately $[F(1,31)=3.88, p<.055]$ than were semantically inconsistent paragraphs. This finding indicates that the subjects were sensitive to the meaning of the paragraphs on the proofreading page, and that their proofreading was disrupted when a semantic inconsistency was encountered. Furthermore, familiar passages were proofread faster than unfamiliar passages $[F(1,31)=41.72, p<.005]$, showing the usual proofreading-time benefit. Unlike the earlier studies, however, there was no main effect of familiarity on proofreading accuracy, although there was a familiarity $X$ consistency interaction $[F(1,31)=4.03, p<.05]$. As Table 4 indicates, the semantic consistency of the passage affected error-detection accuracy only for familiar passages. For these familiar passages, encountering a semantic inconsistency led to a loss in detection accuracy. For the time and accuracy data combined, then, semantic inconsistency slowed proofreading time and decreased proofreading accuracy, indicating awareness of the semantic sense of the proofreading message. Importantly, this consistency effect was just as large when familiar passages were read as when unfamiliar passages were read, with the accuracy data suggesting a trend for greater sensitivity to the message distortion for the familiar passages.

Homograph paragraphs. These paragraphs were also analyzed for the effects of semantic consistency and of

Table 3

Sample Passages Used in Experiment 3

Homonym Passages
(No Pronunciation Shift)
Consistent Version
One night as Bob was walking home, he stopped in front of
the abandoned house at 81 Elmwood Avenue. There was a
strange noise emanating from the dark house. Bob had to ven-
ture in to find what was there. He was terrified; rumor had it
that the house was haunted. He would feel more secure with a
stick to defend himself, and so he went and looked among his
baseball equipment. He found a bat. It was very large and
brown and was to be his protection in the gloomy room*.
Now he didn't need to be afraid any longer.

Inconsistent Version

*It was very large and brown and was flying back and forth in the gloomy room.
Homograph Passages (Pronunciation Shift)

\section{Consistent Version}

The modern day Cinderella stood in front of the mirror and looked sadly at her tattered clothes. She was depressed because she couldn't go to the school dance that night, although she'd hoped for so long to be able to go. Her eyes filled with despair and she began to cry. She looked through the big tears. They would be impossible to disguise even if her makeup dried the wet streaks*. Where would she find a new dress, she wondered? She decided to call her best friend hoping that she might have a dress to lend her for the evening.

\section{Inconsisent Version}

*They would be impossible to disguise even if she mended them with careful stitching. 
Table 4

A. Proofreading accuracy (mean proportion detected) and times (means in seconds) for Experiment 3.

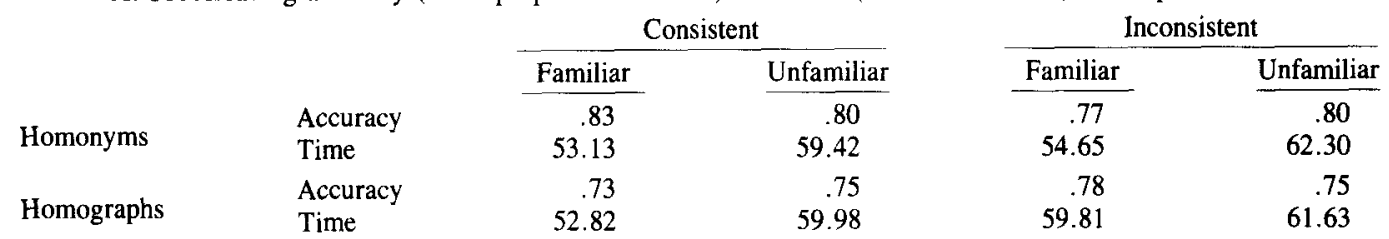

B. Mean Number of correctly recognized sentences per page $(\mathrm{N}=2)$ in Experiment 3.

\begin{tabular}{|c|c|c|c|c|}
\hline & \multicolumn{2}{|c|}{ Consistent } & \multicolumn{2}{|c|}{ Inconsistent } \\
\hline & $\overline{\text { Homographs }}$ & Homonyms & Homographs & Homonyms \\
\hline Familiarized & 1.81 & 1.59 & .75 & .94 \\
\hline Unfamiliarized & 1.59 & 1.47 & .88 & 1.06 \\
\hline
\end{tabular}

familiarity in both the time and accuracy data. The accuracy effects are easily summarized, since neither variable caused any significant effects ( $\mathrm{ps}>.20$ ). Thus, detection accuracy was equivalent for familiar and unfamiliar texts, whether these were semantically consistent or not. The proofreading times, however, showed large effects of both semantic consistency $[\mathrm{F}(1,31)=18.05, \mathrm{p}<.01]$ and of familiarity $[F(1,31)=17.01, p<.005]$. Familiar passages were proofread faster than unfamiliar ones, and semantically consistent texts were proofread faster than inconsistent ones. Importantly, these two variables also entered into a significant interaction $[\mathrm{F}(1,31)=18.87$, $\mathrm{p}<.001]$. As Table 4 indicates, this interaction stemmed from a benefit due to familiarity for the semantically consistent passages $[t(31)=6.45, p<.001]$, a benefit that was lost when the proofreading page contained the inconsistent sentence $[\mathrm{t}(31)=1.33]$. Inconsistent familiar passages were proofread as slowly as unfamiliar passages.

With the time and accuracy data combined, the semantically consistent passages replicate the familiarity effects observed with homonym passages. Familiar passages were proofread faster than unfamiliar passages, with no loss in error-detection accuracy. The loss of an accuracy benefit for familiar passages in this study may be due to the emphasis on comprehending and remembering, but, importantly, these additional task demands did not lead to worse error detection for familiar passages. For the inconsistent passages, another picture emerges. With these, the rereading fluency normally seen for familiar passages was totally erased. The familiar and unfamiliar passages showed no performance differences. It is clear, then, that the subjects must have been sensitive to semantic consistency for these homographic passages, since proofreading speeds were slower for inconsistent than for consistent passages for both familiar and unfamiliar passages. Furthermore, the rereading fluency advantage usually observed for familiar passages was totally lost by the inclusion of one semantically inconsistent phrase on the proofreading page. Thus, the subjects must have been monitoring the proofreading page for meaning repetition, since semantic disruption led to a loss of all familiarity benefits.

Although the semantic-disruption effects for the homograph and homonym passages are slightly different in form, the critical point is that, for both types of passages, the data indicate a sensitivity to the meaning of the proofreading passages for both familiar and unfamiliar texts. The greater "default" effects for familiar than for unfamiliar proofreading texts suggest that the semantic monitoring involved in rapid rereading is, if anything, more disrupted than the semantic processing involved in reading unfamiliar passages. These findings of semanticdistortion effects during proofreading are inconsistent with the visual-scanning view of how texts are proofread. This view would not expect any sensitivity to semantic consistency for proofreading passages, and would certainly not expect greater effects for familiar than for unfamiliar passages. The semantic effects are consistent with a reprocessing-efficiency account.

Sentence recognition. Since the proofreading data clearly indicate sensitivity to meaning of the proofreading texts, the recognition data were examined to see whether these processing disruptions would be mirrored by improved recognition of the disruptive statements. Table 4 contains the mean number $(\mathrm{N}=2)$ of correctly identified critical sentences on each proofreading page. These data were submitted to the same analysis of variance used for the proofreading measures. This analysis indicated a significant effect of semantic consistency $[F(1,31)=$ $71.49, \mathrm{p}<.0001]$, which was qualified by an interaction with passage type $[\mathrm{F}(1,31)=9.41, \mathrm{p}<.005]$. However, an analysis of each passage type separately for the effects of familiarity and semantic consistency found no effects to be statistically reliable. Particularly for the semantically inconsistent trials, recognition was at the chance level. Thus, although the proofreading data indicate sensitivity to these semantic defaults, the additional processing involved in reading the default clause was not sufficient to improve later retention of that clause.

To summarize the effects observed in Experiment 3, the proofreading measures again showed a rereading fluency effect, with no loss in proofreading accuracy, as long as the proofreading passages were semantically consistent with the reading passages. A semantically inconsistent statement disrupted proofreading for both homograph and homonym passages, with both types of passages showing, if anything, greater disruption for familiar than for unfamiliar passages (in accuracy or in time). These 
measures all indicate that the subjects were sensitive to meaning during proofreading, and are consistent with the semantic monitoring view. The scanning view receives no support from these effects.

\section{GENERAL DISCUSSION}

The experiments described here replicate and extend the phenomenon described by Levy (1983). Experiments 1 and 2 demonstrated that errors were detected both faster and more accurately in familiar than in unfamiliar passages. Thus, the potential confounding in the time-limited procedure used in the 1983 studies was not critical to the proofreading facilitation for familiar texts. These two experiments demonstrate that reprocessing fluency (speed gain) is combined with improved, not attenuated, visual processing of the written text, as shown by the superior detection of visual errors within the text.

Experiment 2 also revealed that proofreading speed varied with familiarity with the structure of the passage. Whereas the accuracy effect remained constant over variation in structure, the rereading speed benefit varied with repetition of the previous word arrangement. Thus, normal texts were proofread faster if reading had been of the normal, rather than of a scrambled, version of the text. Similarly, scrambled proofreading was faster if the subjects had previously read that arrangement of scrambled words, rather than the normal text version of those words. Thus, although the familiarity effect observed in the accuracy data could indicate word- or letter-level facilitation, the structural dependency in the speed data indicates that higher order involvement is necessary to explain the entire familiarity benefit on proofreading. Finally, Experiment 3 indicated that proofreaders are sensitive to the semantic sense of the proofreading text, since proofreading performance decreased when the text contained an inappropriate phrase. Furthermore, this disruption in proofreading performance was, if anything, larger, not smaller, for familiar than for unfamiliar texts. Thus, the proofreaders were reading the passages for meaning, not just visually scanning the text for nonword letter combinations, without analyzing the passage's message.

These three studies place several constraints on the possible mechanisms involved in the facilitation observed in proofreading familiar texts. Three explanations will be considered. First, a visual-skills account can be derived from the work of Kolers, Palef, and Stelmach (1980), showing transfer from one reading experience to another when the same visual patterns (in the same typefont and spatial arrangement) were encoded on both occasions. Basically, they suggested that the "priming" of the visual pattern-recognition system during reading led to more rapid rereading when those same visual patterns were reexperienced. According to this logic, the facilitation in proofreading could be due to increased rereading fluency of all text words, combined with a rapid "interrupt" in this fluent visual reprocessing when the deviant letter patterns of the error words were encountered. These "in- terrupts" would lead to accurate identification of the misspelled words, so that proofreading familiar texts should be both faster and more accurate than proofreading unfamiliar texts. In the latter case, the visual operations have not been "primed" by a first reading experience, so neither a fluency gain in rereading nor an interrupt due to misspelling would aid in proofreading. An important aspect of this explanation is that all of the transfer occurs at the level of letter and word patterns, since it is their repetition that accounts for the visual priming effect, with no dependence on higher level processes.

Although the accuracy data from the Levy (1983) studies and from Experiments 1 and 2 showed no variation with text structure, thus suggesting a word- or letterlevel effect, the proofreading-time results of Experiment 2 were clearly inconsistent with this view. In Experiment 2, the four structural-variation conditions were in fact equivalent at the level of word repetition, differing only in the syntactic-semantic organization of the reading and proofreading passages. Therefore, if transfer depended only on word-level repetition, facilitation should have been equivalent in all conditions. The results, however, showed considerable variation in the proofreading-fluency benefit, with variations in the higher order organization. Thus, although the skilled-visual-processing account can address the accuracy effects, it cannot explain this variation in reprocessing fluency due to familiarity. Similarly, the account cannot explain the sensitivity to the semantic distortions in Experiment 3, since "primed" visual operations should be unaffected by semantic deviations. These involvements of higher order processes in the proofreading facilitation, then, suggest that the skilledvisual-processing explanation is insufficient to explain the present data.

Similarly, a visual-scanning account that does not include message analysis is insufficient to account for these findings. Experiment 3 demonstrated that proofreaders were sensitive to the semantic sense of the proofreading message. For both familiar and unfamiliar proofreading tasks, a single phrase that led to a comprehension problem was sufficient to cause a loss in proofreading speed and accuracy. Furthermore, this loss was somewhat greater if the proofreader was familiar with the text. The scanning view cannot account for these findings. The scanning view also fails to account for the specificity effects reported in Experiment 2 and in Levy (1983, Experiment 2). Finally, the scanning view fails to explain why scrambled texts are proofread more slowly than normal texts, since breaking up the syntactic-semantic structure should, if anything, make word scanning easier. Thus, the data cannot be accounted for by a low-level visualscanning explanation, whereby the proofreading text is not read at any higher level of processing.

The data are most consistent with the resource-allocation account proposed by Levy (1983), which predicts higher level involvement in proofreading under some circumstances. The basic idea is that processing resources are normally shared among the data-driven processes involved 
in analyzing the printed words and the conceptually driven processes involved in message understanding. Familiarity with the wording, syntax, or message of a text allows more efficient reanalysis of the text at those levels of processing. This efficient reprocessing might be thought of as more automatic, or less resource demanding, because prior representations of the text are available to facilitate subsequent reprocessing. In this sense, the resource-allocation view is similar to the visual-skills view, except that it involves all levels, not just visual processing.

In applying this notion to the proofreading task, it could be argued that processing resources are insufficient to allow letter-by-letter analyses of the written words, when the proofreader is unfamiliar with the text. Here resources are divided between "seeing" and "understanding," leading to small deviations in the text going undetected. However, familiarity leads to more efficient reprocessing of the words and meaning of the text, thus freeing resources for the proofreading task. These additional resources may be used to read more rapidly, and/or they may be allocated to more detailed letter-by-letter analyses, better meeting the error-detection requirements of the proofreading task. By this view, then, the proofreading facilitation depends on there being less demand for processing resources at the higher text levels. This demand can be decreased when there is familiarity with the text message or when there is no text structure in the proofreading passage (e.g., scrambled text) to demand processing. The resource account, then, differs from the other explanations in giving some importance to the structural arrangement of the proofreading passage.

This resource-allocation view can explain the decreased familiarization benefit under many conditions used in the present experiments. Since meaning is processed during proofreading for both familiar and unfamiliar passages, the sensitivity to semantic distortions found in Experiment 3 would be expected. These meaning distortions recruit resources to the semantic processor, leaving fewer resources for the proofreading task, thus resulting in a loss in proofreading performance. Since the semantic monitoring of familiar messages may be less resource demanding than the semantic processing of unfamiliar messages, more reassignment of resources from proofreading to semantic processing, to deal with the comprehension problem, may occur for familiar than for unfamiliar texts. This greater recruitment of resources from proofreading for familiar texts is consistent with the results of Experiment 3, which indicate greater sensitivity to semantic distortion for familiar texts.

Similarly, the specificity effects found in Experiment 2 can be explained in these terms. When an unfamiliar normal text is proofread, resources are automatically recruited to syntactic-semantic processes. If the proofreader has previously read this text, however, fewer resources will be required to reprocess this "known" message, thus freeing resources to improve proofreading. If the proofreader has been familiarized with the wording, but not the text structure (scrambled reading), however, then resources would again be recruited to syntactic-semantic analyses during proofreading, causing a loss in the familiarization benefit. Thus, the difference in the familiarization effects for normal proofreading, following normal versus scrambled familiarization, is consistent with the resource-allocation account.

The effects observed for the scrambled proofreading conditions, however, are more problematic. Scrambled proofreading should never require resources for higher order processing, since these texts have no structure or message to analyze. However, the absence of structure decreases reading fluency, as shown by slower reading times for scrambled than for normal texts, perhaps indicating more reliance on slower data-driven processing when unrelated words are read. These speed differences for normal versus scrambled texts indicate that this measure is sensitive to higher order dependency during reading. The large familiarity benefit in proofreading speed for the scrambled-scrambled condition suggests that the oral reading experience has made the proofreader familiar with both the word organization and the words themselves, so that during proofreading virtually all resources can go to the proofreading task. This condition may closely parallel visual scanning, without higher order intervention. Even the normal-normal condition requires some resource allocation to semantic monitoring, perhaps explaining the trend toward a smaller benefit there than for the scrambled-scrambled case.

The problem comes, then, in explaining why there is a decreased reprocessing benefit in scrambled proofreading when subjects first read a normal rather than a scrambled version. There is still significant transfer from normal reading to scrambled proofreading, which can be attributed to the lowered demand for higher order processing, as in the normal-normal condition. However, the additional benefit for the scrambled-scrambled case suggests that the oral reading experience made the proofreader more fluent in reading through that particular word arrangement. That is, there is a learned structural effect for scrambled text, somewhat like the syntactic influence on normal reading. This explanation is, of course, highly speculative, since no structural effect had been anticipated for scrambled texts. In sum, then, resource allocation offers an explanation for the higher order effects observed in the present studies, although the scrambled-arrangement effect was surprising.

As a final point, the resource-allocation account also offers an explanation for the decrease in text-specific transfer when the modality, typescript, and wording were changed between reading and proofreading tasks in the Levy (1983) study. As with the semantic changes in Experiment 3 , when the data-driven aspects of the texts were altered from reading to proofreading, resources were reallocated to those levels of processing during proofreading. Consequently, a loss in the proofreading benefit resulted, when resources were drawn to analyze the unfamiliar "data." Thus, the facilitation effect in proofread- 
ing will vary with the similarity between the reading and proofreading texts at both perceptual and conceptual levels. Clearly, the resource-allocation account needs considerable elaboration to predict the magnitude of effects, not just when a disruption of the benefit will occur. For the present, the data indicate a need to consider the flexibility in allocating resources during text processing. These allocations vary considerably with the reader's familiarity with the texts, as well as with the task demands. Reading fluency may be gained by efficiency in processing at any level, so that resources can be reallocated to "needy" processes.

\section{REFERENCES}

Healy, A. F., \& DRewnowski, A. (1983). Investigating the boundaries of reading units: Letter detection in misspelled words. Journal of Experimental Psychology: Human Perception and Performance, 9, 413-426

Kolers, P. A., Palef, S. R., \& Stelmach, L. B. (1980). Graphemic analysis underlying literacy. Memory \& Cognition, 8, 322-328.

LEVY, B. A. (1981). Interactive processing during reading. In A. M. Lesgold \& C. A. Perfetti (Eds.), Interactive processes in reading. Hillsdale, NJ: Erlbaum.

LeVy, B. A. (1983). Proofreading familiar text: Constraints on visual processing. Memory \& Cognition, 11, 1-12.

REICHER, G. M. (1969). Perceptual recognition as a function of meaningfulness of stimulus material. Journal of Experimental Psychology, 81, 274-280.

WheEler, D. (1970). Processes in word recognition. Cognitive Psychology, 1, 59-85.

(Manuscript received March 28, 1984; revision accepted for publication July 23, 1984.) 